\title{
Unser Gesundheitswesen im internationalen Vergleich
}

\begin{abstract}
Im Verhältnis zum Bruttoinlandprodukt hatte $\mathbf{2 0 0 6}$ die Schweiz nach den USA wiederum das zweitteuerste Gesundheitswesen aller 30 OECD-Länder. Gemessen an den Pro-Kopf-Ausgaben war es das drittteuerste System. Mit ihrer finanziellen Direktbelastung der Kranken ist die Schweiz einsam an der Spitze. Die Ärztedichte ist überdurchschnittlich hoch. Auf einer neuen CD der OECD finden sich auch Daten über Krankenhäuser und zahllose weitere Statistiken.
\end{abstract}

Gerhard Kocher

1 OECD Health Data 2008. Statistics and indicators for 30 countries. Paris: OECD Publishing; 2008. CD-ROM mit Benutzerhandbuch, sechssprachig englisch/französisch/ deutsch/italienisch/spanisch/ russisch. www.oecd.org/health/ healthdata.

2 Empfehlenswert ist auch das 110-seitige Heft «World Health Statistics 2008». Das Themenspektrum ist kleiner, erfasst sind aber 193 Länder. Genf: WHO; 2008. www.who.int/whosis/ whostat/en. Französische Version: fr statt en.

Korrespondenz:

Dr. rer. pol. Gerhard Kocher

Haldenweg $10 \mathrm{~A}$

CH-3074 Muri b. Bern

Tel. 0319526707

gerhard.kocher@muri-be.ch
Im Juni hat die OECD (Organisation für wirtschaftliche Zusammenarbeit und Entwicklung, Paris) ihre jährlich aktualisierte CD mit umfangreichen Daten über die Gesundheitswesen ihrer 30 Mitgliedländer publiziert [1]. Sie enthält über 1200 Indikatoren mit Zeitreihen, die bis 1960 zurückgehen. Die neuesten Zahlen betreffen meist das Jahr 2006. Die CD ist die zuverlässigste Sammlung von Gesundheitsdaten über die 30 Länder [2]. Sie beruht auf den Arbeiten von Hunderten von Statistikern in den Mitgliedsländern und in Paris. Die 660000 Daten betreffen Gesundheitszustand, Ressourcen und Inanspruchnahme, Medikamente, Ausgaben und Finanzierung, soziale Sicherheit, nichtmedizinische Gesundheitsfaktoren sowie demographische und wirtschaftliche Entwicklung. Damit ist die CD eine wahre Datenbonanza.

\section{Starke Zunahme der Pro-Kopf-Ausgaben in sechs Jahren}

Von 2000 bis 2006 nahmen im OECD-Durchschnitt die kaufkraftbereinigten Gesundheitsausgaben pro Kopf um 49\% zu (Tab. 1). Gering war die Zunahme in Japan (26\%), Deutschland und Österreich, mittelstark in Schweden, Frankreich, in den Niederlanden, in Kanada, in den USA, in Belgien, Norwegen und Grossbritannien. Eine starke Kostenentwicklung erlebten Südkorea (97\%), die Slowakei, Ungarn, Griechenland, Irland und Luxemburg. Die Schweiz liegt mit 32,4\% Wachstum ein Drittel unter dem OECDDurchschnitt.

Im Ausgabenvergleich 2006 allerdings ist die Schweiz mit 4311 US-\$ im dritten Rang und 53\% über dem OECD-Mittel. Wie immer sind die USAusgaben exorbitant, nämlich 2,4-mal höher als der Durchschnitt und 49\% höher als das zweitplazierte Norwegen. Bemerkenswert tief sind weiterhin die britischen Ausgaben (41,1\% der US-
Tabelle 1

Gesundheitsausgaben pro Kopf in US-\$ (kaufkraftbereinigt).

\begin{tabular}{|c|c|c|c|}
\hline Land & 2000 & 2006 & $\mathrm{Zu}-/ \mathrm{Abnahme}$ in $\%$ \\
\hline USA & 4570 & 6714 & 46,9 \\
\hline Norwegen & 3039 & 4520 & 48,7 \\
\hline Schweiz (b) & 3256 & 4311 & 32,4 \\
\hline Luxemburg (b) & 2554 & 4303 & 68,5 \\
\hline Kanada & 2513 & 3678 & 46,4 \\
\hline Österreich & 2859 & 3606 & 26,1 \\
\hline Belgien & 2377 & 3488 & 46,7 \\
\hline Frankreich & 2421 & 3449 & 42,5 \\
\hline Niederlande & 2337 & 3391 & 45,1 \\
\hline Deutschland & 2671 & 3371 & 26,2 \\
\hline Dänemark & 2379 & 3349 & 40,8 \\
\hline Island & 2736 & 3340 & 22,1 \\
\hline Schweden & 2284 & 3202 & 40,2 \\
\hline Irland & 1801 & 3082 & 71,1 \\
\hline Australien (a) & 2265 & 2999 & 32,4 \\
\hline Grossbritannien & 1847 & 2760 & 49,4 \\
\hline Finnland & 1794 & 2668 & 48,7 \\
\hline Italien & 2053 & 2614 & 27,3 \\
\hline Griechenland & 1429 & 2483 & 73,8 \\
\hline Japan (a) & 1967 & 2474 & 25,8 \\
\hline Spanien & 1536 & 2458 & 60 \\
\hline Neuseeland (b) & 1604 & 2448 & 52,6 \\
\hline Portugal & 1509 & 2120 & 40,5 \\
\hline Ungarn & 852 & 1504 & 76,5 \\
\hline Tschechien & 980 & 1490 & 52 \\
\hline Südkorea & 753 & 1480 & 96,5 \\
\hline Slowakei (a) & 603 & 1130 & 87,4 \\
\hline Polen & 583 & 910 & 56,1 \\
\hline Mexiko & 508 & 794 & 56,3 \\
\hline Türkei (a) & 432 & 591 & 36,8 \\
\hline Durchschnitt & 1950 & 2824 & 49,2 \\
\hline
\end{tabular}

a Spalte 2006: Zahl für 2005.

b Spalte 2006: Schätzung 
Ausgaben). $\mathrm{Zu}$ ausgedehnten und oft hitzigen Diskussionen führt seit längerem der Vergleich USA/Kanada: Wie kann das Nachbarland im Norden ein qualitativ vergleichbares Gesundheitssystem mit fast nur den halben Kosten der USA betreiben? Im Vergleich zu 2005 konnten nur Island und Irland ihre Ausgaben geringfügig senken. In allen anderen Ländern stiegen sie, am stärksten in den USA um 367 und in der Schweiz um 242 US-\$.

Vergleicht man die nationalen Ausgaben für das Gesundheitswesen nicht kaufkraftbereinigt, sondern zum Wechselkurs, sind die Pro-KopfAusgaben der USA nur 14,2\% höher als die der Schweiz (5877 bzw. 6714 US-\$).

\section{Schweiz wiederum im zweiten Rang der Gesundheitsausgaben in Prozent des BIP} Im internationalen Vergleich ist eine zweite Rankingmethode wichtig, die den Wohlstand der Länder berücksichtigt. Bei diesem Vergleich werden die Gesundheitsausgaben in Prozent des Bruttoinlandprodukts (BIP) ausgedrückt (Tab. 2). Nichts geändert hat sich hier in den beiden Spitzenrängen: In allen Jahren seit 1999 sind die USA mit weitem Abstand an der Spitze, immer gefolgt von der Schweiz, wenn auch mit grossem Abstand, der seit 2000 noch leicht gewachsen ist. 2006 lagen die US-Ausgaben 71,9\% über dem OECD-Durchschnitt, die schweizerischen 27\%. Die kanadischen Ausgaben sind auch in diesem Vergleich nur halb so hoch wie die der USA. Grossbritannien hat den OECD-Durchschnitt von $8,9 \%$ des BIP immer noch nicht erreicht, obwohl die Angleichung seit einigen Jahren ein erklärtes Ziel der Regierung ist. Der Abstand ist etwa gleich gross wie 2000.

Im Vergleich des Ausgabenwachstums seit 2000 ist die Schweiz im 23. Rang der 30 OECDLänder: 9,7\% gegenüber 14,5\%. Bei den Vergleichen in diesem Abschnitt ist zu berücksichtigen, dass die Zahlen der Tabelle 2 nicht nur auf den Gesundheitsausgaben beruhen, sondern auch auf der wirtschaftlichen Entwicklung (gemessen am Bruttoinlandprodukt per capita). Dieses stieg von 2000 bis 2006 im Schnitt um 77\%, von Land zu Land aber höchst unterschiedlich. Die Extreme sind Japan (-7\%) und Slowakei (+170\%). Unter dem Durchschnitt liegen unter anderen die Schweiz mit 50\% und die USA mit nur 27\% Wachstum. Aussagekräftige Ländervergleiche müssen sich deshalb immer auf die Zahlen beider Tabellen stützen.

Die OECD-CD enthält keine Kostenprognosen. Für die Schweiz existiert eine aktuelle Studie [3] über die Kostenentwicklung bis 2030. Je nach

\section{Tabelle 2}

Nationale Gesundheitsausgaben 2006 in \% des Bruttoinlandprodukts BIP.

\begin{tabular}{|c|c|c|c|}
\hline Land & 2000 & 2006 & $\mathrm{Zu}$-/Abnahme in \% \\
\hline USA & 13,2 & 15,3 & 15,9 \\
\hline Schweiz & 10,3 & 11,3 & 9,7 \\
\hline Frankreich & 9,6 & 11,1 & 15,6 \\
\hline Deutschland & 10,3 & 10,6 & 2,9 \\
\hline Belgien & 8,6 & 10,4 & 20,9 \\
\hline Portugal & 8,8 & 10,2 & 15,9 \\
\hline Österreich & 9,9 & 10,1 & 2,0 \\
\hline Kanada & 8,8 & 10,0 & 13,6 \\
\hline Dänemark & 8,3 & 9,5 & 14,5 \\
\hline Niederlande & 8,0 & 9,3 & 16,3 \\
\hline Neuseeland & 7,7 & 9,3 & 20,8 \\
\hline Schweden & 8,2 & 9,2 & 12,2 \\
\hline Island & 9,5 & 9,1 & $-4,2$ \\
\hline Griechenland & 7,8 & 9,1 & 16,7 \\
\hline Italien & 8,1 & 9,0 & 11,1 \\
\hline Australien (a) & 8,3 & 8,8 & 6,0 \\
\hline Norwegen & 8,4 & 8,7 & 3,6 \\
\hline Spanien & 7,2 & 8,4 & 16,7 \\
\hline Grossbritannien & 7,2 & 8,4 & 16,7 \\
\hline Ungarn & 6,9 & 8,3 & 20,3 \\
\hline Japan (a) & 7,7 & 8,2 & 6,5 \\
\hline Finnland & 7,0 & 8,2 & 17,1 \\
\hline Irland & 6,3 & 7,5 & 19,0 \\
\hline Luxemburg & 5,8 & 7,3 & 25,9 \\
\hline Slowakei (a) & 5,5 & 7,1 & 29,1 \\
\hline Tschechien & 6,5 & 6,8 & 4,6 \\
\hline Mexiko & 5,6 & 6,6 & 17,9 \\
\hline Südkorea & 4,6 & 6,4 & 39,1 \\
\hline Polen & 5,5 & 6,2 & 12,7 \\
\hline Türkei (a) & 4,9 & 5,7 & 16,3 \\
\hline Durchschnitt & 7,8 & 8,9 & 14,5 \\
\hline
\end{tabular}

(a) Spalte 2006: Zahl für 2005

Szenario werden im Jahr 2030 die Gesundheitsausgaben 14,4 oder 17,2\% des Bruttoinlandprodukts betragen, was 111 oder 123 Milliarden Franken (zu konstanten Preisen von 2004) entspricht. Zukunftsbezogen ist auch eine Studie vom Juli 2008 über die ambulante Versorgung bis 2030 [4].

\section{Höhere Gesundheitsausgaben, höhere Lebenserwartung?}

Die Abbildung 1 belegt einmal mehr die Erkenntnis, dass Lebenserwartung und Gesundheitsausgaben nicht parallel laufen. Japan als Land mit der höchsten Lebenserwartung (83 Jahre) ist bei den Gesundheitsausgaben nur im Rang 23 von 
Abbildung 1

Nationale Gesundheitsausgaben in Prozent des BIP (Skala links) und Lebenserwartung in Jahren (Skala rechts) 2006.

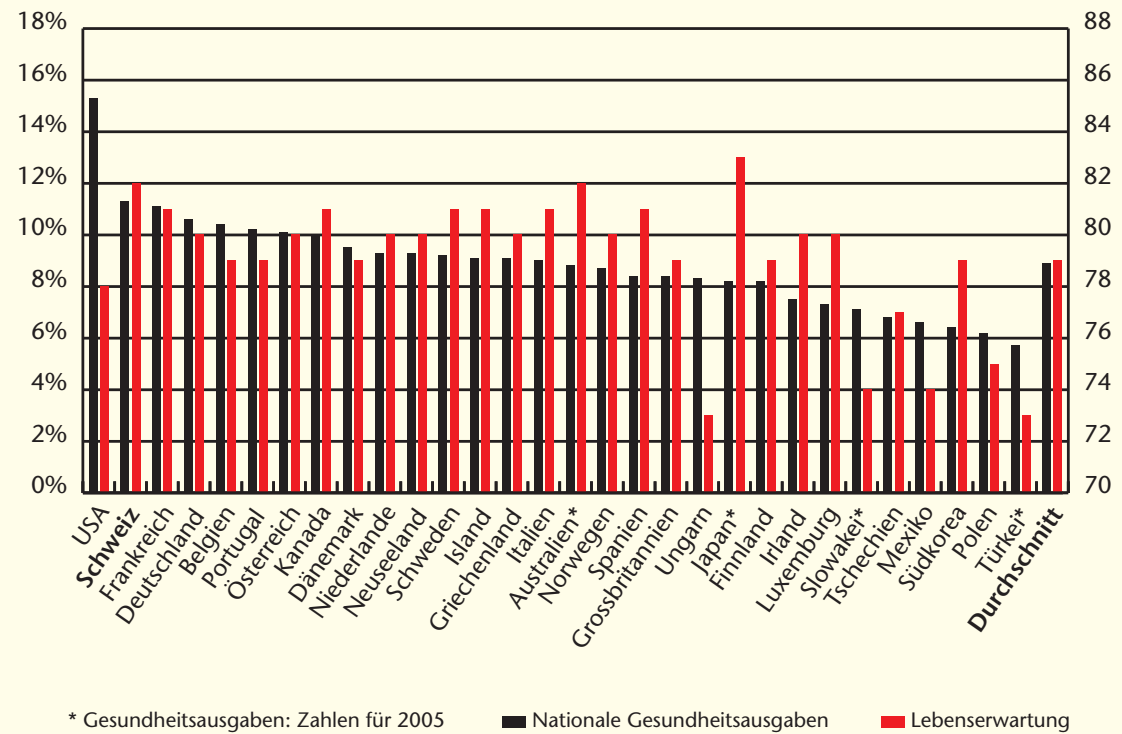

5 Réformes et régulation des systèmes de santé en Europe. Revue française des affaires sociales. 2006;60;1-375.

6 Tanner M. The grass is not always greener. A look at national health care systems around the world. Policy Analysis (Cato Institute, Washington D.C.) 2008:613;1. www.cato.org/pub_display.php? pub_id=9272.

7 Peterson CL, Burton R. U.S. health care spending: Comparison with other OECD countries. Congressional Research Service, Washington D.C. 2007. http://assets.opencrs.com/rpts/ RL34175_20070917.pdf.

8 Schoen C, et al. Toward higherperformance health systems: Adults' health care experiences in seven countries. Health Affairs, Web Exclusive. 2007;26: w717-w734. http://content. healthaffairs.org/cgi/content/full/ 26/6/w717.

9 Why not the best? Results from the National Scorecard on U.S. Health System Performance, 2008. New York: The Commonwealth Fund; 2008.

www.commonwealthfund.org/ publications/publications_show. htm?doc_id=692682.
30 Ländern. 82 Jahre erreicht die Schweiz mit Rang 2 der Gesundheitsausgaben. Allerdings erzielt Australien im Rang 16 das gleiche Resultat. Einmal mehr völlig aus dem Rahmen fallen die USA. Mit den weitaus höchsten Gesundheitsausgaben aller 30 OECD-Länder weisen sie eine Lebenserwartung von nur 78 Jahren aus. Schlechter schneiden nur sechs Länder ab, deren Gesundheitsausgaben aber im Schnitt nicht einmal halb so hoch sind wie der USA-Rekordwert. Viele andere Daten auf der OECD-CD legen ähnliche Diskrepanzen offen. Deutlich belegen sie eine oft ignorierte Selbstverständlichkeit: Die Effektivität eines Gesundheitssystems hängt nicht in erster Linie von der Höhe der Gesundheitsausgaben ab, sondern viel stärker von einer Fülle anderer Faktoren. Dies zeigen auch ein ausgezeichneter Vergleich (375 Seiten) der europäischen Gesundheitssysteme [5] und drei Ländervergleiche aus den USA [6-8]. Ein Zwei-Jahres-Vergleich des USGesundheitswesens ergab, dass trotz Mehrausgaben von rund 240 Milliarden US-\$ ein auf 37 Kriterien basierender Leistungs- und Qualitätsscore in diesen zwei Jahren gesunken ist [9]. Solche Erkenntnisse sollten Anlass sein, die zunehmende «Heiligsprechung der Gesundheitsausgaben» (s. dazu [10]) zu bekämpfen.

\section{Erklärungsfaktoren für unterschiedliche nationale Gesundheitsausgaben}

Wer ist schuld an der «Kostenexplosion»? Warum sind die Kostenniveaus in vergleichbaren Ländern so unterschiedlich? Dies sind zwei Grundfragen, die in der Gesundheitsökonomie seit 30 Jahren immer wieder erörtert werden. Klare Antworten scheitern an der Datenlage, an politischen Interessen und vor allem daran, dass die Ausgaben multifaktoriell bedingt sind. Ohne Mühe lassen sind 50 und mehr Faktoren aufzählen, die die Ausgabenhöhe eines Landes positiv oder negativ beeinflussen. Behauptungen auf Stammtischniveau können bestenfalls für einige Ursachen zutreffen, nicht aber für Dutzende von anderen relevanten Faktoren. Die Gewichtung der einzelnen Faktoren ist zusätzlich ein kaum zu lösendes Problem.

Paul Camenzind vom Obsan hat praktisch zeitgleich mit der OECD-CD eine aufschlussreiche Studie «Erklärungsansätze regionaler Kostenunterschiede im Gesundheitswesen» publiziert [11]. Sie befasst sich nicht nur mit den intranationalen (hauptsächlich kantonalen), sondern auch mit den internationalen Kostenunterschieden. Dazu hat Camenzind 24 international vergleichende Studien analysiert. Statistisch signifikante Erklärungsfaktoren sind danach die Höhe des Bruttoinlandprodukts pro Kopf (wichtigster Faktor), die Altersstruktur, der Urbanisierungsgrad, die Angebotsstruktur (z.B. Ärztedichte), Finanzierungsmechanismen und andere Elemente. Die Ergebnisse zur theoretischen Umschreibung des Kostengeschehens seien allerdings «ziemlich bescheiden». Camenzind: «Unterschiede beim Aufbau, der Organisation und der Finanzierung der Gesundheitssysteme führen [...] oft zu enormen Schwierigkeiten, welche fast nicht überwunden werden können.»

\section{«Weltmeister» in der Direktbelastung der Kranken}

Seit jeher zahlen die Schweizer im internationalen Vergleich die weitaus höchsten Beträge aus dem eigenen Geldbeutel (Tab. 3). 2006 waren es geschätzte 1779 US-\$ pro Kopf, mehr als doppelt so viel wie die USA (857 US-\$) und über dreimal mehr als der Durchschnitt der erfassten 26 Länder (504 US-\$). Diese Out-of-pocket-Zahlungen betreffen vor allem die rezeptfreien Medikamente, Pflegeheime, Spitex und in der Schweiz praktisch die ganze Zahnmedizin (über 3 Milliarden Franken/Jahr).

Mit der Direktbelastung in der Schweiz (1779 US-\$/Kopf) werden 30,3\% der nationalen Ausgaben für das Gesundheitswesen finanziert. Im OECD-Durchschnitt sind es 18,9\%. Mit ihrer hohen Selbstzahlungsquote ist unser Land im dritten Rang der 26 Länder. Nur in Mexiko $(52,3 \%)$ und Südkorea $(36,8 \%)$ ist die Quote 
Tabelle 3

Selbstzahlungen (Privathaushalte) pro Kopf, US-\$, zu Wechselkurs.

\begin{tabular}{|c|c|c|c|}
\hline Land & 2000 & 2006 & Zu-/Abnahme in \% \\
\hline Schweiz (b) & 1174 & 1779 & 52 \\
\hline Norwegen & 528 & 977 & 85 \\
\hline Island & 557 & 901 & 62 \\
\hline USA & 684 & 857 & 25 \\
\hline Dänemark & 396 & 697 & 76 \\
\hline Österreich & 397 & 648 & 63 \\
\hline Finnland & 344 & 603 & 75 \\
\hline Australien (a) & 342 & 579 & 69 \\
\hline Italien & 377 & 572 & 52 \\
\hline Kanada & 329 & 567 & 72 \\
\hline Spanien & 244 & 512 & 110 \\
\hline Deutschland & 266 & 491 & 85 \\
\hline Irland & 173 & 483 & 179 \\
\hline Südkorea & 211 & 435 & 106 \\
\hline Portugal & 215 & 424 & 97 \\
\hline Japan (a) & 477 & 415 & -13 \\
\hline Neuseeland (b) & 162 & 400 & 147 \\
\hline Luxemburg (b) & 154 & 318 & 106 \\
\hline Mexiko & 167 & 275 & 65 \\
\hline Frankreich & 156 & 265 & 70 \\
\hline Niederlande & 172 & 227 & 32 \\
\hline Ungarn & 86 & 209 & 143 \\
\hline Polen & 74 & 142 & 92 \\
\hline Slowakei (a) & 22 & 141 & 541 \\
\hline Tschechien & 35 & 109 & 211 \\
\hline Türkei (a) & 54 & 76 & 41 \\
\hline Durchschnitt & 312 & 504 & 62 \\
\hline
\end{tabular}

a Spalte 2006: Zahl für 2005

b Spalte 2006: Schätzung

10 Kocher G. Heiligsprechung der Gesundheitsausgaben? Schweiz Ärztezeitung. 2008;89;(1-2):24-5.

11 Camenzind P. Erklärungsansätze regionaler Kostenunterschiede im Gesundheitswesen. Analyse der internationalen gesundheitsökonomischen und gesundheitsstatistischen Literatur - mit besonderem Fokus auf die Schweiz. Neuenburg: Obsan; 2008 www.bfs.admin.ch/bfs/portal/de/ index/infothek/publ.Document. 110309.pdf.

12 Oggier W, Kocher G (Hrsg.) Gesundheitswesen Schweiz 2007-2009. Bern: Hans Huber; 2007. Version française: Oggier W, Kocher G (éds.). Système de santé suisse 2007-2009. Berne: Hans Huber; 2007. höher. Unsere 30,3\% sind erheblich höher als u.a. die $20,2 \%$ in Italien, die $16,5 \%$ in Österreich, die 13,2\% in Deutschland und die 12,8\% in den USA. Ganz am Schluss der Liste finden sich Frankreich mit 6,7\% und die Niederlande mit $6 \%$.

\section{Ärztedichte überdurchschnittlich hoch}

Die schweizerische Ärztedichte ist seit Jahren rund ein Viertel höher als der OECD-Durchschnitt (Tab. 4). Mit den Niederlanden war die Schweiz im Jahr 2006 im dritten Rang der 28 erfassten Länder. Zwischen 2000 und 2006 ist die Ärztedichte nur in Italien, Finnland und Ungarn gesunken. Im Schnitt aller Länder stieg sie um 11,9\% (Schweiz: 8,6\%). Sehr starke Zunahmen von über $27 \%$ verzeichneten Luxemburg, Irland, Grossbritannien, Südkorea und Norwegen.
Die Statistiken zur Ärztedichte sind allerdings weniger zuverlässig als andere OECD-Daten. Dies gilt weniger für die nationalen Zeitreihen als für internationale Vergleiche. Eine wichtige Ursache der grossen Unterschiede von Land zu Land sind unterschiedliche Definitionen des «berufstätigen» bzw. «praktizierenden» Arztes. Diese Differenzen werden aber auf der CD in umfangreichen Anmerkungen zu den Tabellen ausgewiesen. Ein ungelöstes Problem bei Ärztedichtezahlen sind die von Arzt zu Arzt sehr unterschiedlichen Aktivitätsgrade und Arbeitspensen von 5 bis 100 (oder auch 200!) Prozent. Ohne ihre Berücksichtigung sind blosse Kopfzahlen von beschränkter Aussagekraft für den Ist-Zustand und besonders für Prognosen.

Generell ist zu bedauern, wie wenig statistische Daten über Mediziner und Arztpraxen (und auch Zahnarztpraxen) vorliegen. Dies betrifft nicht nur, aber speziell auch die Schweiz. Einer der wichtigsten und auch teuersten Sektoren der Gesundheitsversorgung ist eine Statistikwüste mit nur wenigen Oasen. Von Jahr zu Jahr verbessert sich die Datenlage nur wenig. Riesige Datenmengen bei Verbänden, Ämtern, Versicherungen, Firmen der Praxisadministration, TrustCentern und Qualitätserhebern sind vorhanden, aber kaum erschlossen und wenig zugänglich. Als Mitherausgeber (mit Willy Oggier) von bisher drei Auflagen des Buches «Gesundheitswesen Schweiz - Eine aktuelle Übersicht» [12] bin ich immer wieder enttäuscht, dass in den sieben Jahren von der ersten Auflage bis heute nur minime Fortschritte $\mathrm{zu}$ verzeichnen sind. Bezeichnend ist auch, dass es noch heute kein einziges Buch gibt, das die ambulante Versorgung unseres Landes ausführlich darstellt - und das bei einer wahren Bücherflut zu Medizin und Gesundheitsversorgung.

\section{Spürbarer Wandel \\ bei den Krankenhäusern}

Für die Bettendichte liegen die Daten für 25 der 30 OECD-Länder vor (Tab. 5). Die Schweiz hatte 2006 pro 1000 Einwohner deutlich weniger Krankenhausbetten als der OECD-Durchschnitt, nämlich 3,5 gegenüber 5,5 Betten. Die Bettendichte in Deutschland, Österreich und Frankreich ist gemäss OECD mehr als doppelt so hoch. Die Schweizer Bettendichte 2006 entspricht dem 20. Rang von 25 Ländern. Im Jahr 2000 waren wir im 15., 1995 noch im 10. Rang. Der Bettenabbau 1995-2006 belief sich im OECD-Durchschnitt auf $11 \%$, in der Schweiz auf $36 \%$.

Die durchschnittliche Akutbettenbelegung 2006 in der Schweiz ist hoch: $86,8 \%$. Nur in Kanada 
Tabelle 4

Berufstätige Ärzte pro 1000 Einwohner, 1995, 2000, 2006. Index: Durchschnitt aller Länder im betreffenden Jahr = Index 100 .

\begin{tabular}{|c|c|c|c|c|c|c|c|}
\hline Land & 1995 & Index & 2000 & Index & 2006 & Index & Zu-/Abnahme 2000-2006 \\
\hline Griechenland (c) & 3,9 & 150 & 4,3 & 154 & 5 & 167 & 16,3 \\
\hline Belgien & 3,5 & 135 & 3,9 & 139 & 4 & 133 & 2,6 \\
\hline Niederlande (b) & & & 3,2 & 114 & 3,8 & 127 & 18,8 \\
\hline Schweiz & 3,2 & 123 & 3,5 & 125 & 3,8 & 127 & 8,6 \\
\hline Island & 3 & 115 & 3,4 & 121 & 3,7 & 123 & 8,8 \\
\hline Italien & 3,9 & 150 & 4,1 & 146 & 3,7 & 123 & $-9,8$ \\
\hline Norwegen & 2,8 & 108 & 2,9 & 104 & 3,7 & 123 & 27,6 \\
\hline Spanien & 2,5 & 96 & 3,2 & 114 & 3,6 & 120 & 12,5 \\
\hline Tschechien & 3 & 115 & 3,4 & 121 & 3,6 & 120 & 5,9 \\
\hline Österreich & 2,7 & 104 & 3,1 & 111 & 3,6 & 120 & 16,1 \\
\hline Deutschland & 3,1 & 119 & 3,3 & 118 & 3,5 & 117 & 6,1 \\
\hline Schweden (c) & 2,9 & 112 & 3,1 & 111 & 3,5 & 117 & 12,9 \\
\hline Frankreich & 3,2 & 123 & 3,3 & 118 & 3,4 & 113 & 3 \\
\hline Portugal (c) & 2,9 & 112 & 3,1 & 111 & 3,4 & 113 & 9,7 \\
\hline Ungarn & 3 & 115 & 3,1 & 111 & 3 & 100 & $-3,2$ \\
\hline Irland & 2,1 & 81 & 2,2 & 79 & 2,9 & 97 & 31,8 \\
\hline Australien (c) & 2,5 & 96 & 2,5 & 89 & 2,8 & 93 & 12 \\
\hline Luxemburg & 2,2 & 85 & 2,1 & 75 & 2,8 & 93 & 33,3 \\
\hline Finnland & 2,4 & 92 & 2,8 & 100 & 2,7 & 90 & $-3,6$ \\
\hline Grossbritannien & 1,8 & 69 & 1,9 & 68 & 2,5 & 83 & 31,6 \\
\hline USA & 2,2 & 85 & 2,3 & 82 & 2,4 & 80 & 4,3 \\
\hline Neuseeland & 2 & 77 & 2,2 & 79 & 2,3 & 77 & 4,5 \\
\hline Polen & 2,3 & 88 & 2,2 & 79 & 2,2 & 73 & 0 \\
\hline Japan (a) & & & 1,9 & 68 & 2,1 & 70 & 10,5 \\
\hline Kanada & 2,1 & 81 & 2,1 & 75 & 2,1 & 70 & 0 \\
\hline Mexiko & 1,7 & 65 & 1,6 & 57 & 1,9 & 63 & 18,8 \\
\hline Südkorea & 1,1 & 42 & 1,3 & 46 & 1,7 & 57 & 30,8 \\
\hline Türkei & 1,1 & 42 & 1,3 & 46 & 1,6 & 53 & 23,1 \\
\hline Durchschnitt & 2,6 & & 2,8 & & 3 & & 11,9 \\
\hline
\end{tabular}

Tabelle 5

Krankenhausbetten insgesamt, Zahl je 1000 Einwohner.

\begin{tabular}{lcccr} 
Land & $\mathbf{1 9 9 5}$ & $\mathbf{2 0 0 0}$ & $\mathbf{2 0 0 6}$ & Zu-/Abnahme 2000-2006 \\
\hline Japan & 15,4 & 14,7 & 14 & $-4,8$ \\
\hline Südkorea & 4,4 & 6,1 & 8,5 & 39,3 \\
\hline Deutschland & 9,7 & 9,1 & 8,3 & $-8,8$ \\
\hline Tschechien & 9,1 & 8,5 & 8,2 & $-3,5$ \\
\hline Ungarn & 8,8 & 8,1 & 7,9 & $-2,5$ \\
\hline Österreich & 9,3 & 8,6 & 7,6 & $-11,6$ \\
\hline Belgien * & 7,4 & 7,8 & 7,4 & $-5,1$ \\
\hline Frankreich & 8,9 & 8,1 & 7,2 & $-11,1$ \\
\hline Finnland & & 7,5 & 6,9 & -8 \\
\hline Slowakei & & 7,8 & 6,7 & $-14,1$ \\
\hline Irland * & 7 & 6,3 & 5,6 & $-11,1$ \\
\hline
\end{tabular}


Tabelle 5

(Fortsetzung)

\begin{tabular}{llllc} 
Land & $\mathbf{1 9 9 5}$ & $\mathbf{2 0 0 0}$ & $\mathbf{2 0 0 6}$ & Zu-/Abnahme 2000-2006 \\
Griechenland * & 4,9 & 4,7 & 4,7 & 0 \\
\hline Niederlande & 5,3 & 5,2 & 4,5 & $-13,5$ \\
\hline Italien & 6,3 & 4,7 & 4 & $-14,9$ \\
\hline Australien * & 4,6 & 4 & 3,9 & $-2,5$ \\
\hline Dänemark * & 4,9 & 4,3 & 3,7 & -14 \\
\hline Norwegen & 4 & 3,8 & 3,6 & $-5,3$ \\
\hline Portugal * & 4,1 & 3,9 & 3,6 & $-7,7$ \\
\hline Grossbritannien & 4,8 & 4,1 & 3,6 & $-12,2$ \\
\hline Schweiz & 5,5 & 4,1 & 3,5 & $-14,6$ \\
\hline Kanada * & 5 & 3,8 & 3,4 & $-10,5$ \\
\hline Spanien * & 4,3 & 3,7 & 3,4 & $-8,1$ \\
\hline USA & 4,1 & 3,5 & 3,2 & $-8,6$ \\
\hline Türkei & 2,5 & 2,6 & 2,7 & 3,8 \\
Mexiko & 1,9 & 1,8 & 1,7 & $-5,6$ \\
Durchschnitt & 6,2 & 5,9 & 5,5 & $-6,1$
\end{tabular}

* 2006: Zahl für 2005

(91\%) und Norwegen (88,5\%) ist sie noch höher. Im Schnitt der erfassten 22 OECD-Länder beträgt sie $75,7 \%$. Am tiefsten ist die Belegungsrate in Mexiko (60,5\%) und den Niederlanden (63,9\%). Von 2000 bis 2006 verlief die Entwicklung der Bettenbelegung uneinheitlich: In 13 Ländern nahm sie zu (in der Schweiz um 2,3\%), in 8 Ländern ging sie zurück. Insgesamt stieg die Belegung in den 21 Ländern, für die die betreffenden Daten vorliegen, in den 6 Jahren nur um $0,6 \%$. Die höhere Auslastung der Bettenkapazitäten in der Schweiz ist zum Teil erklärbar mit dem starken Bettenabbau (14,6\%) in der gleichen Zeitperiode.

Die Zahl der Krankenhaustage eines Landes ist für die Kostenentwicklung eine zentrale Grösse. Die 1,1 Akutspitaltage pro Einwohner und Jahr in der Schweiz entsprechen genau dem Durchschnitt der erfassten 22 OECD-Länder (Tab. 6). Über 50\% mehr Tage verbringen die Deutschen und Österreicher in Krankenhäusern der Akutversorgung. Andererseits gibt es zahlreiche Länder mit gut ausgebauter Gesundheitsversorgung, die nur 0,9 oder weniger Aufenthaltstage verzeichnen. Dass die völlig unterschiedlichen Gesundheitssysteme der Niederlande und der USA den gleichen, extrem tiefen Wert von 0,7 Tagen aufweisen, ist ein Indiz mehr, dass einfach gestrickte, wenn möglich noch monokausale Erklärungsversuche im Gesundheitssystem fehl am Platz sind.

\section{Tabelle 6}

Krankenhaustage in der Akutversorgung pro Einwohner. Index: Durchschnitt 2006 = Index 100.

\begin{tabular}{lllll} 
Land & $\mathbf{1 9 9 5}$ & $\mathbf{2 0 0 0}$ & $\mathbf{2 0 0 6}$ & Index \\
\hline Japan & 3 & 2,4 & 2 & 182 \\
\hline Österreich & 2 & 1,9 & 1,8 & 164 \\
\hline Deutschland & 2,2 & 2 & 1,7 & 155 \\
\hline Tschechien & 2 & 1,7 & 1,7 & 155 \\
\hline Ungarn & 2 & 1,7 & 1,5 & 136 \\
\hline Luxemburg & & 1,4 & 1,3 & 118 \\
\hline Belgien (a) & 1,4 & 1,4 & 1,2 & 109 \\
\hline Slowakei & & 1,5 & 1,2 & 109 \\
\hline Schweiz & 1,7 & 1,3 & 1,1 & 100 \\
\hline Australien (a) & 1,1 & 1 & 1 & 91 \\
\hline Frankreich & 1,3 & 1,1 & 1 & 91 \\
\hline Finnland (a) & 1,1 & 0,9 & 0,9 & 82 \\
\hline Irland (a) & 1 & 0,9 & 0,9 & 82 \\
\hline Italien (a) & 1,3 & 1,1 & 0,9 & 82 \\
\hline Kanada (a) & 1,1 & 1 & 0,9 & 82 \\
\hline Norwegen & 1 & 0,9 & 0,9 & 82 \\
\hline Grossbritannien & 0,8 & 0,9 & 0,9 & 82 \\
\hline Portugal (a) & 0,8 & 0,9 & 0,8 & 73 \\
\hline Spanien (a) & 0,8 & 0,8 & 0,8 & 73 \\
\hline Niederlande & 1 & 0,8 & 0,7 & 64 \\
\hline USA & 0,8 & 0,7 & 0,7 & 64 \\
\hline Mexiko & 0,4 & 0,4 & 0,4 & 36 \\
\hline Durchschnitt & $\mathbf{1 , 3}$ & $\mathbf{1 , 2}$ & $\mathbf{1 , 1}$ & 100 \\
\hline & & & & \\
\hline
\end{tabular}

a 2006: Zahl für 2005 